
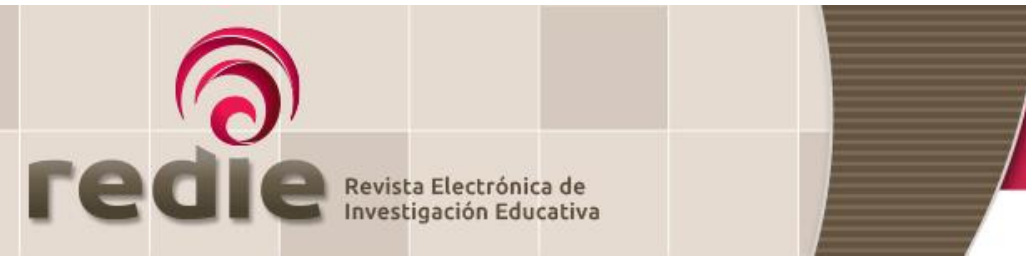

Vol. 20, Núm. 4, 2018

\title{
Mentoría en educación superior, la experiencia en un programa extracurricular
}

\author{
Mentoring in Higher Education, Experience in an Extracurricular \\ Program
}

\author{
Mónica Irene Camacho Lizárraga (*) monica.camacho@cide.edu \\ (*) Centro de Investigación y Docencia Económicas \\ (Recibido: 29 de mayo de 2017; Aceptado para su publicación: 26 de junio de 2017)
}

Cómo citar: Camacho, I. (2018). Mentoría en educación superior, la experiencia en un programa extracurricular. Revista Electrónica de Investigación Educativa, 20(4), 86-99. https://doi.ora/10.24320/redie.2018.20.4.1999

\section{Resumen}

Este artículo analiza posibles contribuciones de la mentoría a la formación de universitarios en un programa extracurricular. Primero se aborda el concepto de mentoría y sus conceptualizaciones teóricas, y luego se describe el estudio de caso: la mentoría en un programa extracurricular de liderazgo para estudiantes universitarios con habilidades cognitivas sobresalientes (Programa Talentum-Universidad), así como la metodología utilizada -basada en el análisis de los reportes que mentores y mentorandos entregaron después de la primera y última sesión. Los resultados de la investigación dan evidencia de la contribución del proceso de mentoría a la formación de los estudiantes en el ámbito personal, académico y profesional, subrayando la importancia de las metas de interés del mentorando y las características o atributos del mentor, abonando a la escasa investigación sobre esta práctica en educación superior.

Palabras clave: Mentoría, estudiantes universitarios, actividad extracurricular, desarrollo integrado.

\begin{abstract}
This article analyzes the potential contributions of mentoring to the formative process of college students in an extracurricular program. The first section deals with the concept of mentoring and contemporary theoretical conceptualizations. The second section describes the case study a mentoring component within an extracurricular leadership program for college students with outstanding cognitive skills (Talentum-Universidad Program). It also describes methodology used, consisting in the analysis of the reports that mentors and mentees submitted for the first and last mentoring session. The final section presents the findings; based on the evidence, mentoring contributes to the formative process of college students in three areas: personal, academic and professional. The mentee's goals and the characteristics of the mentor are highlighted as key factors. In sum, the document adds to the existing but scarce research about mentoring practices in higher education.
\end{abstract}

Keywords: Mentoring, college students, extracurricular activities, integrated development. 


\section{Introducción}

La figura mentor-mentorando data de tiempos ancestrales, por sus aportaciones como modalidad de enseñanza-aprendizaje para el desarrollo de conocimientos y habilidades. En sistemas educativos escolarizados y no escolarizados aprendientes de diferentes edades y niveles de desarrollo han participado en diferentes formas de mentoría.

Como resultado de la masificación en el acceso a la educación superior en las últimas décadas, los programas de mentoría han ganado espacios en las instituciones educativas; sin embargo, aunque el primer estudio sobre mentoría en educación superior data de 1911 por parte de la Facultad de Ingeniería de la Universidad de Michigan (Johnson, 1989), es escasa la investigación sobre esta práctica en este nivel (Clark, 1994; Knippelmeyer y Torraco, 2007).

Este artículo busca aportar al conocimiento sobre la mentoría en educación superior al responder a la pregunta principal de investigación: ¿Contribuye la mentoría al proceso formativo de universitarios en un programa extracurricular?; y a la pregunta secundaria: de ser así, ¿cuál es la principal contribución?

Para responder a estas preguntas se utilizó la metodología de estudio de caso, analizando el componente de mentoría dentro de un programa extracurricular para estudiantes universitarios con habilidades cognitivas sobresalientes. El análisis de datos consistió en una codificación cualitativa de los reportes proporcionados por los mentores y estudiantes participantes.

\subsection{Conceptualizaciones sobre mentoría}

Estudios en investigación educativa reportan diferentes razones que impulsan prácticas formales de mentoría en educación superior, entre ellas: apoyar procesos formativos (por ejemplo, elevar niveles de logro académico), promover aspectos de identidad individual o grupal, facilitar la transición entre estados de desarrollo, y reducir o evitar la deserción. Hallazgos de la Psicología Aplicada coinciden en que intervenciones modeladas como esas buscan generar un efecto en la conducta del participante, efectos que se pueden obtener por diferentes métodos, pero que guardan en común mecanismos de tipo cognitivo.

Bozeman y Feeney (2007) reportan la ausencia de una teoría robusta para investigar la mentoría; mientras que otros investigadores señalan las dificultades para conformar una definición (Anderson y Shannon, 1988; Gehrke, 1988; Healy y Welchert, 1990) y la escasez de modelos formales para llevarla a cabo (Burke y McKeen, 1997), lo que denota que el desarrollo de la literatura sobre el tema "se encuentra en una etapa temprana de desarrollo" (DuBois y Karcher, 2005, p. 4).

Se han documentado esfuerzos en aras de una definición unificada, por ejemplo, Jacobi (1991) identificó 15 definiciones al analizar campos disciplinares como Educación, Psicología y Administración. Roberts (2000) analizó literatura publicada entre 1978 y 1999, documentando atributos esenciales y contingentes sobre mentoría. Un estudio más reciente, focalizado en administración, teoría organizacional y educación para el trabajo, resultó en 11 definiciones (Bozeman y Feeney, 2007, p. 273), lo que demuestra una falta de consenso incluso en un mismo campo disciplinar.

Compilaciones como las elaboradas por Dubois y Karcher (2005) y Eby, Rhodes y Allen (2010) han contribuido a delinear atributos en común de prácticas de mentoría que permiten establecer el marco de referencia a utilizar en el presente análisis (tabla I). 
Tabla I. Atributos comunes de la mentoría

\begin{tabular}{|c|c|}
\hline $\begin{array}{l}\text { Dubois y Karcher } \\
(2005, \text { p. } 3)\end{array}$ & $\begin{array}{l}\text { - El mentor es alguien con mayor experiencia o juicio que el mentorando. } \\
\text { - El mentor ofrece guía o instrucción con la intención de facilitar el crecimiento y } \\
\text { desarrollo del mentorando. } \\
\text { - Hay un lazo emocional entre el mentor y el mentorando, en el cual un sello } \\
\text { característico es la confianza. }\end{array}$ \\
\hline $\begin{array}{l}\text { Eby, Rhodes y Allen } \\
(2010, \text { p. 10) }\end{array}$ & $\begin{array}{l}\text { - Es una relación única entre los individuos. Ninguna mentoría es igual, distintos } \\
\text { intercambios interpersonales y patrones de interacciones idiosincráticas definen y } \\
\text { moldean la relación. } \\
\text { - Es una alianza de aprendizaje. Aunque las metas de la relación de mentoría pueden } \\
\text { diferir a lo largo de locaciones y relaciones, casi toda mentoría involucra la adquisición } \\
\text { de conocimiento. Aprendizajes ocurren independientemente de la calidad de las } \\
\text { relaciones y de las expectativas de las partes involucradas. } \\
\text { - Es un proceso definido por los tipos de apoyo que el mentor proporciona al } \\
\text { mentorando; mientras que las funciones específicas que caracterizan a la mentoría } \\
\text { varían, son ampliamente clasificadas como emocionales o psicosociales (por ejemplo, } \\
\text { de amistad, aceptación, apoyo) e instrumentales o relacionadas con la profesión (por } \\
\text { ejemplo, de acompañamiento, información, de apoyo o recomendación). } \\
\text { - Es una relación recíproca pero asimétrica. Aunque el mentor podría beneficiarse de la } \\
\text { relación, el objetivo principal es el crecimiento y desarrollo del mentorando. } \\
\text { - Es una relación dinámica: cambian en el tiempo y el impacto de la mentoría se } \\
\text { incrementa con el paso del mismo. }\end{array}$ \\
\hline
\end{tabular}

Otros trabajos han señalado lo que la mentoría no es, e incluso hacen una crítica del concepto mismo. Roberts (2000) identificó atributos contingentes al fenómeno de la mentoría, tales como: coaching, modelado de roles, evaluación informal y patrocinio (sponsorship); mientras que Rísquez (2008) es puntual al señalar la confusión que suele existir entre mentoría y conceptos como tutoría, coaching y consejería, por lo que ofrece la siguiente guía (tabla II).

Tabla II. Qué es y qué no es la mentoría

\begin{tabular}{l|l}
\hline Mentoría es... & Mentoría no es... \\
\hline $\begin{array}{l}\text { Una mejora de otras formas de apoyo social, emocional, } \\
\text { psicológico e intelectual. }\end{array}$ & Una solución aislada a problemas. \\
\hline $\begin{array}{l}\text { Un proceso dinámico que compromete a ambos, mentor y } \\
\text { mentorando, al autoaprendizaje, acción y reflexión. }\end{array}$ & Algo que se le hace a un individuo. \\
\hline $\begin{array}{l}\text { Transformacional, orgánica, compleja, multidimensional y de } \\
\text { alguna forma impredecible. Requiere de mutuo compromiso. }\end{array}$ & Pasiva o mecanicista. \\
\hline
\end{tabular}

Fuente: Rísquez (2008, p. 63)

Una diferencia importante entre la tutoría y la mentoría es que en la primera el objetivo es que el estudiante domine un área de conocimiento, de tal forma que el experto asigna un problema, evalúa el desempeño y durante el proceso suele tener control de los problemas que plantea al estudiante. Mientras que en la segunda, el enfoque de la interacción gira en torno a los problemas que plantea el participante considerado "junior" (O'Neill y Harris, 2005, p. 113).

Por su parte, Hall (2003) señala que la ideología detrás de la mentoría como concepto no ha escapado a las críticas. En especial, sobre la cercanía entre ésta y el mundo de los negocios, cuestionando -ademásen qué proyectos o temas son mentorados realmente los individuos y si al final del día es una actividad que reproduce el statu quo (Gulam y Zulfiqar, 1998), por lo que recomiendan atraer al proceso a mentores con un amplio rango de antecedentes. 
Entre las definiciones propuestas se revisaron las siguientes:

Es una relación protegida en la cual aprendizaje y experimentación pueden ocurrir, habilidades potenciales ser desarrolladas, y en la cual los resultados pueden medirse en términos de competencia ganada más que por el territorio curricular cubierto. (Gibbons, 2000, p. 18).

Es una relación de no-parentesco entre un adulto de mayor edad y experiencia con un joven protégé, en la cual el adulto proporciona de manera continua guía, instrucción y aliento para el desarrollo de la competencia y carácter del protégé." (Rhodes, 2002, p. 3).

Además de las proporcionadas por Anderson y Shannon (1988), Gehrke (1988), Healy y Welchert (1990), y Yamamoto (1988). Para fines de este artículo la definición de referencia es la de Garringer, Kupersmidt, Rhodes, Stelter y Tai (2015):

La mentoría tiene lugar entre personas jóvenes y personas de mayor edad o con mayor experiencia quienes actúan o trabajan en una capacidad no profesional para establecer una relación basada en el apoyo que beneficie una o más áreas de desarrollo del mentorando. (Garringer et al., 2015, p. 9)

Es posible observar la evolución que con relativa rapidez presentan las definiciones de mentoría. Un ejemplo es la utilizada por MENTOR/National Mentoring Partnership: ${ }^{1}$

Mentoría es una relación estructurada y de confianza que reúne a gente joven con personas que se preocupan por ellos ofreciendo guía, apoyo y aliento con el objetivo de desarrollar competencias y carácter en el mentorando. (MENTOR/National Mentoring Partnership, 2003, como se cita en DuBois y Karcher, 2015, p. 4)

\section{Estudio de caso: la mentoría en Talentum-Universidad}

Talentum-Universidad es un programa extracurricular de liderazgo para estudiantes universitarios con habilidades cognitivas sobresalientes. Diseñado e implementado por el Programa Interdisciplinario sobre Política y Prácticas Educativas (PIPE) del Centro de Investigación y Docencia Económicas, A. C. (CIDE), con dos ediciones concluidas a la fecha $\left(2015\right.$ y $2016^{2}$ ) con una duración de 5 meses cada una.

Como intervención educativa, Talentum-Universidad tiene por objetivo identificar jóvenes universitarios con habilidades cognitivas y de liderazgo sobresalientes, e interés por el compromiso social; y contribuir a la formación y fortalecimiento de habilidades clave en su crecimiento académico, profesional y personal. Además, Talentum-Universidad busca promover el encuentro y colaboración de jóvenes talentosos provenientes de distintas regiones y estratos sociales del país, e impulsar la formación meritocrática con compromiso social de redes entre los participantes, egresados y aliados del programa. En el largo plazo, el programa busca ofrecer evidencia para sustentar la formulación de una política educativa nacional que identifique y apoye a los jóvenes universitarios con talento intelectual y habilidades de liderazgo.

Talentum-Universidad inicia cada año en el mes de marzo y consta de cinco etapas: a) semana de inducción; b) trabajo en equipo y a distancia, para lo cual se les asigna un reto a resolver; c) viaje internacional; d) práctica "sombra"; y e) competencia final de proyectos (ver Anexo 1). Además, los estudiantes participan en el componente de mentoría, objeto de análisis de esta investigación, en el cual se les asigna un mentor externo que les brindará acompañamiento y asesoría a lo largo del programa. A continuación se explica el proceso de selección a Talentum-Universidad y el componente de mentoría para los estudiantes admitidos al programa.

\footnotetext{
${ }^{1}$ Organización estadounidense sin fines de lucro fundada hace 25 años, cuya misión es alimentar la calidad y cantidad de relaciones de mentoría para los jóvenes de Estados Unidos y cerrar la brecha de que existe en mentoría para uno de cada tres jóvenes que crecen sin este apoyo crítico.

${ }^{2}$ La edición 2015 la financió la Subsecretaría de Educación Superior (SES), de la Secretaría de Educación Pública (SEP); mientras que la edición 2016, la SES y Santander Universidades, programa de responsabilidad de Banco Santander.
} 


\subsection{Proceso de selección al programa Talentum-Universidad}

Estudiantes universitarios mexicanos de todo el país son invitados a participar por medio de una convocatoria abierta, publicada y difundida en medios digitales. Los estudiantes que cumplen con el perfil solicitado ${ }^{3}$ participan en un proceso de selección en tres fases, que inicia en la plataforma tecnológica del programa con su registro en línea -en el que proporcionan la documentación solicitada ${ }^{4}$ toman una prueba de razonamiento lógico. Los estudiantes que superan la línea de corte de esa prueba y cuentan con un registro completo avanzan a la segunda fase, en la que presentan un total de cinco pruebas adicionales: tres de habilidades cognitivas (razonamiento matemático, verbal, de apego a la tarea) y dos de habilidades no cognitivas. Aquellos estudiantes cuyos resultados superan la línea de corte establecida en el puntaje ponderado, que integra el puntaje de las pruebas, se convierten en finalistas y avanzan a la última fase del proceso en la que sostienen dos entrevistas vía Skype. ${ }^{5}$

Solicitaron admisión 11,000 estudiantes en la edición 2015 y 13,000 estudiantes en la edición 2016. En cada edición se seleccionaron 60 universitarios, 30 hombres y 30 mujeres, quienes integraron la primera y segunda generación del programa. En el Anexo 2 se presenta una ficha descriptiva con las características generales de los integrantes de cada generación.

\subsection{La mentoría}

En Talentum-Universidad, la mentoría tiene por objetivo proporcionar al estudiante una estructura de apoyo a partir del acompañamiento y asesoría que le brinda un mentor externo, en dos ejes temáticos principalmente: 1) Futuro académico y profesional, es decir, sus posibles planes a futuro ya sea estudiar un posgrado, incorporarse al mercado laboral u otras posibilidades; y 2) Reto asignado a trabajar en equipo.

Inicialmente la mentoría se introdujo en el programa como "piloto" en la primera edición en 2015, presentándola a los estudiantes como un recurso de apoyo y seguimiento en los temas antes descritos y de participación opcional, por lo que se inscribieron 46 de 60 jóvenes. En la edición 2016 se realizaron los cambios siguientes a este componente: se presentó a los estudiantes como un componente formal del programa (de participación ya no opcional); un total de cinco sesiones mentor-mentorando a lo largo del programa (en el piloto de 2015 fueron tres sesiones); y requiere que al menos una de las sesiones sea en formato presencial (en el piloto todas fueron a distancia); el reporte a entregar a la coordinación del programa después de cada sesión -tanto el mentor, como el mentorando- se llena en línea (en el piloto el formato a llenar fue enviado por correo electrónico).

Salvo las puntuales diferencias ya mencionadas entre ambas ediciones, el diseño del componente de mentoría consiste en lo siguiente:

1. Proceso de asignación. Al inicio de Talentum-Universidad a cada participante se le asigna un mentor(a) externo, con base en afinidades entre ambos, ya sea por campo disciplinar o intereses laborales; ambas partes son informadas sobre el resultado de la asignación.

\footnotetext{
${ }^{3}$ Ser estudiante mexicano de cualquier carrera inscrito en las instituciones de educación superior de México, tanto públicas como privadas; cursar del 5o. semestre en adelante de la licenciatura; ser alumno regular con promedio mínimo de 8.0 y no tener más de un año de haber terminado de cursar materias; poseer liderazgo, motivación, compromiso e interés en contribuir al desarrollo de México.

${ }^{4}$ Currículum vítae; carta de recomendación académica por parte de un profesor con quien el estudiante haya cursado clases o trabajado en actividades de asesoría o investigación (con rúbrica e información de contacto); carta de exposición de motivos en la que el estudiante explique por qué considera poseer los atributos de liderazgo, motivación y compromiso para participar en este programa (máximo 1000 palabras); constancia de estudios que indique estatus como alumno regular, el promedio acumulado y con membrete o sello de la institución; y un video original de auto presentación del candidato con duración máxima, de dos minutos específicamente para la convocatoria de este programa.

${ }^{5}$ En la edición 2016, además de las dos entrevistas, los estudiantes desarrollaron un ensayo sobre un tema asignado.
} 
2. Intercambio de información mentor-mentorando. Al mentor se le proporciona información sobre el estudiante que será su mentorando (carta de motivos y currículum vítae), y al estudiante, información sobre el perfil profesional de su mentor (formato con información de contacto y curriculum vitae).

3. Capacitación sobre la metodología. Tanto al mentor como al mentorando se les capacita utilizando los documentos informativos y materiales preparados para conducir el proceso.

a) Antes de iniciar el proceso se entrega a ambos una guía en la que se describe qué es y qué no es la mentoría, se reiteran los ejes temáticos a tratar en las sesiones y la duración de éstas (30 minutos máximo). Se les proporciona también un código de ética, el cual busca garantizar un manejo cuidadoso de lo tratado en las sesiones de mentoría y mantener el trabajo dentro de los ejes temáticos establecidos. Finalmente, se explica a ambos la utilización del formato que cada uno deberá llenar en línea reportando los avances de cada sesión, y el calendario de inicio y término. Las sesiones se programan de común acuerdo, pero es el mentorando quien debe tomar la iniciativa de contactar al mentor para programar las cinco sesiones (de las cuales una deberá ser presencial, durante la semana de inducción o de clausura de Talentum-Universidad).

b) Como parte de la metodología, previo a la primera sesión, el mentorando deberá identificar un máximo de tres metas a trabajar durante las sesiones. Lo que permite dar un sentido de enfoque y dirección al proceso, y a la coordinación del programa evaluar el avance sobre tales metas al concluir la mentoría.

c) Al término de la última sesión, la coordinación del programa evalúa el avance del estudiante con base en las metas que él/ella estableció, y analiza los comentarios cualitativos del mentor.

\subsection{Perfil de los mentores}

Los mentores son invitados a participar de manera voluntaria, sin recibir percepción económica alguna, y seleccionados con base en un perfil de trayectoria profesional destacada en su campo de actividad, incluyendo representación de los siguientes sectores: academia, función pública, iniciativa privada y organizaciones no gubernamentales (ONG); en la edición 2015 participaron 27 mentores para atender a 46 estudiantes, y en el 201645 mentores atendieron a 60 estudiantes. ${ }^{6}$ Cabe mencionar que entre una edición y otra, la composición del grupo de mentores invitados presenta cambios, en función del perfil de la generación Talentum-Universidad que se trate. Por ejemplo, en la primera edición hubo una mayor proporción de estudiantes matriculados en Ciencias Sociales; mientras que en la segunda edición hubo una mayor proporción de estudiantes matriculados en ingenierías. En el grupo de mentores voluntarios para la segunda edición hubo una tasa de repetición del $27 \%$, es decir, que ya habían sido mentores en la primera edición del programa.

\section{Resultados}

Se realizó un análisis de contenido a lo reportado en los formatos entregados después de realizar las sesiones de mentoría, lo que permitió codificar las ideas más frecuentes y agruparlas por afinidad generando categorías. Posteriormente éstas fueron ordenadas de mayor a menor, según la frecuencia de las ideas reportadas. A continuación se presentan los hallazgos principales que los mentores reportaron de sus mentorandos en los siguientes aspectos: metas a trabajar en la mentoría; avance en las metas propuestas; tipo de metas en que el estudiante logró avanzar, y posteriormente se muestran los hallazgos sobre tres aspectos reportados por los estudiantes: beneficios obtenidos de la mentoría y desempeño del mentor (acciones y características).

\footnotetext{
${ }^{6}$ En la edición 2016 participaron 45 mentores: 16 se dedican a la academia de tiempo completo, 16 se desempeñan en la iniciativa privada, 9 en la función pública y 4 en ONG.
} 
Metas a trabajar en la mentoría por el estudiante, según el mentor. De manera general, las metas a trabajar que los estudiantes expresaron en su primera sesión se mantienen a lo largo del componente. Una proporción mínima (8.3\% en la edición 2016 del programa) reporta haber cambiado alguna de las metas, o incluso haber incorporado alguna nueva a lo largo del proceso por considerarlo de utilidad en virtud del perfil o experiencia del mentor.

En ambas ediciones del programa las metas que los estudiantes se propusieron trabajar en la mentoría se mantienen en mayor medida dentro de los ejes temáticos definidos. Figuran de manera preponderante temas relacionados con el futuro laboral (definir objetivos laborales, identificar empresas u organizaciones en donde emplearse o para realizar prácticas profesionales; emprender una empresa propia) y con el futuro académico (posgrado a estudiar, becas, movilidad internacional, establecer una línea de investigación, tema de tesis, voluntariado, servicio social, publicar un artículo). Y en menor medida otros como: aprender de la experiencia del mentor (sobre su área disciplinar de sus estudios profesionales, trayectoria, campo de actividad); y abordar dudas sobre el programa (proyecto a trabajar en equipo y viaje al extranjero, por mencionar algunos).

Un hallazgo que destaca es un eje temático que no había sido considerado en los lineamientos de la mentoría, y que en la edición 2016 del programa recibió el mayor número de menciones por parte de los estudiantes: obtener de su mentor retroalimentación sobre habilidades propias, con la finalidad de desarrollar un perfil más competitivo; o como lo expresó en el reporte un estudiante, para "saber si voy por el buen camino". La tabla III muestra el tipo de metas a trabajar en la mentoría, reportadas de mayor a menor frecuencia por los estudiantes en cada edición de Talentum-Universidad.

Tabla III. Tipo de metas a trabajar en la mentoría reportadas por los estudiantes

\begin{tabular}{l|l}
\hline Edición 2015 & Edición 2016 \\
\hline 1. Futuro laboral. & $\begin{array}{l}\text { 1. Retroalimentación sobre habilidades propias. } \\
\text { 2. Futuro académico. }\end{array}$ \\
$\begin{array}{ll}\text { 2. Futuro académico. } \\
\text { 4. Temas relacionados con el programa. }\end{array}$ & $\begin{array}{l}\text { 3. Futuro laboral. } \\
\text { 4. Aprender sobre la experiencia del mentor. }\end{array}$ \\
\hline
\end{tabular}

Avance en las metas propuestas por el estudiante. De manera consistente, en ambas ediciones de Talentum-Universidad el 93\% de los mentores reportó que su mentorando logró un avance significativo en las metas que se planteó trabajar en la mentoría. En la edición 2015 del programa, el 7\% de los mentores indicó que no hubo avance; mientras que en la edición 2016, el 4\% de los mentores reportó un avance parcial y el 3\% restante no respondió.

Estos resultados son similares a lo reportado por los mentorandos: 90\% considera que sí logró un avance significativo en las metas que se planteó para este componente; $8 \%$ reporta un avance parcial; y $2 \%$ respondió que no. ${ }^{7}$

Tipo de meta en que el estudiante logró avanzar, según el mentor. Los mentores identificaron un mayor avance por parte de sus mentorandos en dos tipos de metas, las relacionadas con el futuro laboral (empleabilidad, por ejemplo) y con el futuro académico (estudios de posgrado). Los mentores coinciden en señalar un menor avance en metas sobre el desarrollo de habilidades y actitudes propias, tales como: seguridad en sí mismo; autoconocimiento; establecer prioridades; capacidad de enfoque; receptividad y apertura. Un menor avance puede explicarse debido a que metas de esta naturaleza implican un proceso de desarrollo personal, que implica un mayor tiempo a cinco meses (duración de la mentoría), en comparación a metas de otro tipo, como tomar una decisión a futuro sobre el tipo de posgrado a estudiar, identificar el tipo de organización en la cual realizar prácticas profesionales o en la cual emplearse. La tabla IV muestra, de mayor a menor frecuencia, el tipo de metas reportadas por el mentor en las que el estudiante logró un mayor avance.

\footnotetext{
${ }^{7}$ Datos disponibles sólo para la edición 2016.
} 
Tabla IV. Tipo de metas en las que el mentorando logró mayor avance

\begin{tabular}{l|l}
\hline Edición 2015 & Edición 2016 \\
\hline 1. Futuro laboral & 1. Futuro académico \\
2. Habilidades propias & 2. Futuro laboral \\
& 3. Habilidades propias \\
\hline
\end{tabular}

A continuación se presentan los hallazgos sobre tres aspectos reportados ${ }^{8}$ por los estudiantes: beneficios obtenidos de la mentoría, desempeño del mentor y características del mentor.

Beneficios. Al preguntar a los estudiantes si consideran haber logrado sus metas en la mentoría, ${ }^{9}$ en primer lugar (41\% de las respuestas) afirman haber adquirido una mayor claridad sobre los temas tratados; en segundo lugar ( $35 \%$ de las respuestas) por la retroalimentación y consejos recibidos del mentor; en tercer lugar (11\% de las respuestas) porque la mentoría les dio mayor preparación para fortalecer sus habilidades o realizar actividades específicas; en cuarto lugar (8\%) por haber logrado mayor seguridad en sí mismo y para tomar decisiones, y en quinto lugar (5\%) por haber recibido orientación en proyectos específicos, como emprendimiento social o de negocios (figura 1).



Figura 1. Beneficios obtenidos de la mentoría

Desempeño del mentor. Acciones que los estudiantes consideran importantes. Sobre las acciones que más valoran de su mentor, se mencionan en orden de importancia: ${ }^{10}$ que el mentor busque crear un ambiente de confianza (21 menciones); que sea un escucha activo, "que muestre interés en lo que yo le estoy contando" (18 menciones); que tenga apertura para compartir "conocimientos o saberes" (12 menciones); y disposición para compartir "experiencias" (12 menciones). Otras mencionadas con menor frecuencia fueron: que el mentor ayude a "crear un plan, a definir u organizar ideas" (8 menciones); que responda preguntas o explique con claridad (8 menciones); que brinde consejos (7 menciones); y en el mismo orden de importancia, que el mentor haga preguntas específicas (2 menciones) y encomiende tareas (2 menciones) (ver Fig. 2)

\footnotetext{
${ }^{8}$ Datos disponibles sólo para la edición 2016 del programa.

9 De las múltiples respuestas que podían dar los estudiantes sólo se consideró la primera respuesta.

${ }^{10}$ La información se presenta en número de menciones y no de porcentajes. El número de menciones es mayor al número de participantes (60) debido a que cada estudiante podía dar más de una respuesta.
} 


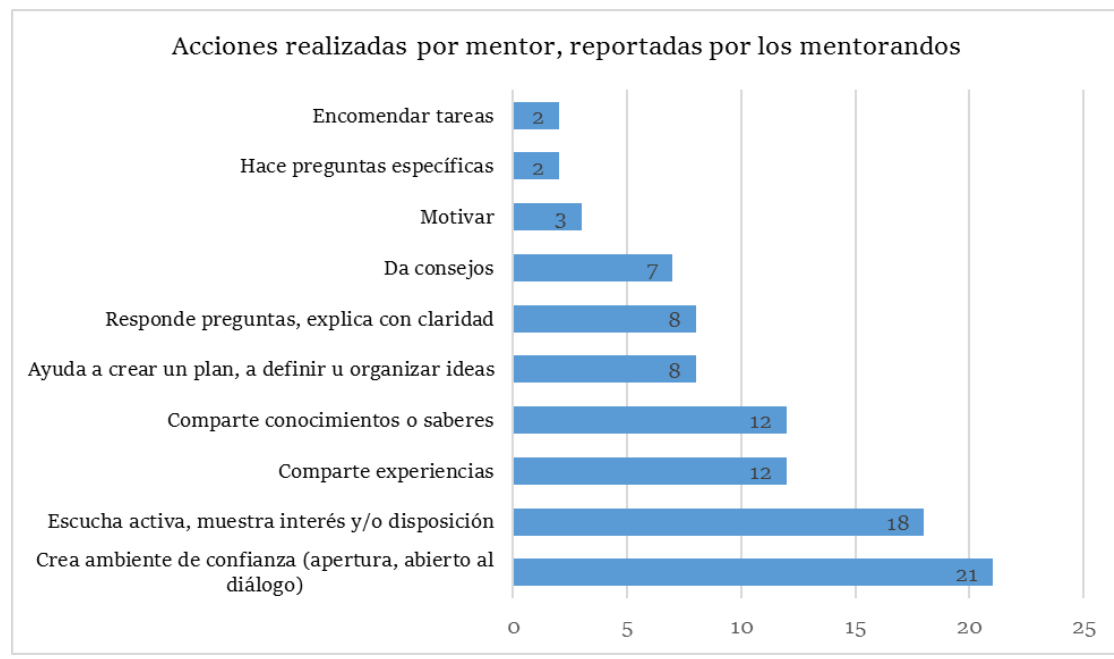

Figura 2. Desempeño del mentor según los mentorandos

Características del mentor. Lo que a los estudiantes les pareció más importante en su mentor es, principalmente: que tenga una actitud abierta o disposición (18 menciones); que tenga una actitud positiva (9 menciones); que sea amable (7 menciones); que muestre atributos como "sencillez, accesibilidad, paciencia y humildad" (7 menciones); que tenga capacidad de orientar (7 menciones); que muestre atributos como "franqueza, sinceridad" que sea "directo" en su comunicación" (3 menciones); que sea proactivo, con iniciativa (3 menciones); que sea "altruista, generoso", que busque ayudar (3 menciones). Estas respuestas se muestran en forma gráfica en la figura 3.

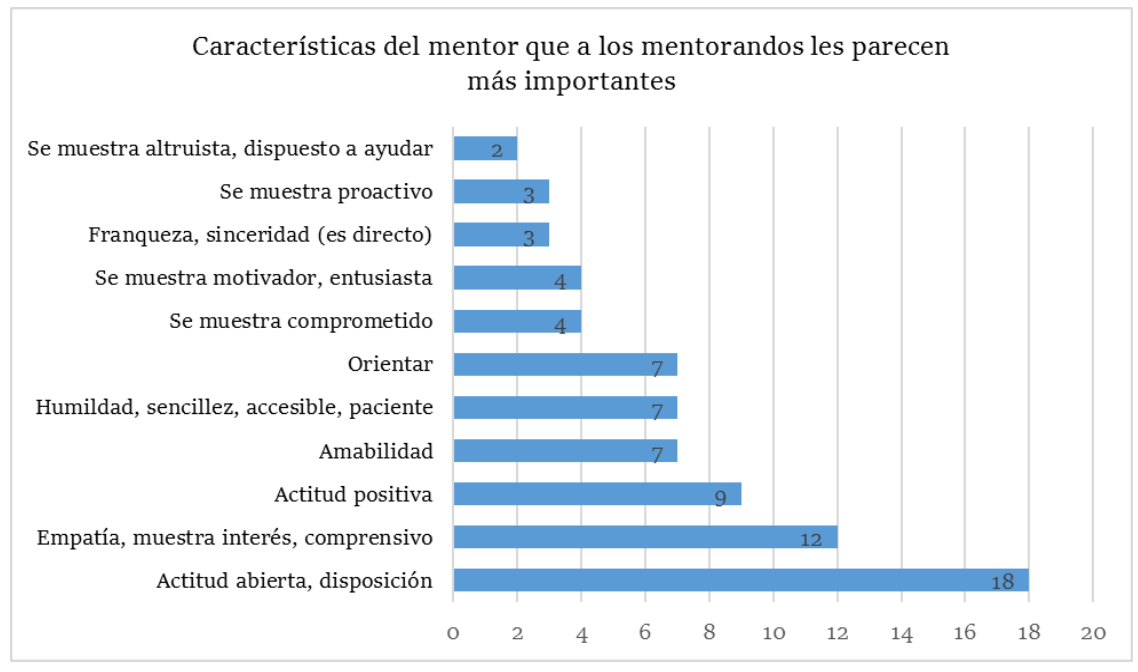

Figura 3. Características del mentor más importantes para los mentorandos

\section{Conclusiones}

El estudio de caso sobre la mentoría en el programa Talentum-Universidad proporciona evidencia para afirmar que sí contribuye al proceso formativo de los estudiantes de este programa extracurricular.

De manera consistente, el grupo de mentores voluntarios en cada edición del programa respondió que su mentorando sí logró un avance en las metas que se propuso (93\% de los mentores en cada edición); o 
incluso en menor proporción (4\%) reportó un avance parcial. ${ }^{11}$ Por parte de los estudiantes, es muy similar la tasa de respuesta sobre su sentido de logro en la mentoría: el 90\% considera que sí logró un avance significativo en las metas que se planteó. ${ }^{12}$

La contribución de la mentoría para los jóvenes que actualmente están cursando el 50\% o más de su plan de estudios es de dos tipos, pero también hay otras contribuciones dependiendo de cada mentoría, pues cada una es una experiencia única. Coles (2011, p. 2) afirma que "la mentoría sirve a diferentes propósitos, especialmente basados en la edad y necesidades del individuo"; mientras que Eby et al. (2010, p. 10) señalan sobre la mentoría que, "es una relación única entre los individuos. Ninguna mentoría es igual, distintos intercambios interpersonales y patrones de interacciones idiosincráticas definen y moldean la relación".

Sin embargo, con base en el análisis de la evidencia presentada, surgen dos contribuciones principales para el estudiante: 1) Como espacio en sí misma para la exploración de ideas y para recibir apoyo de diferentes tipos, y 2) Como una alianza de aprendizaje externa al aula.

La mentoría representa un espacio para la exploración de intereses, preocupaciones, y desarrollar aspectos de identidad individual y colectiva. Para que ese espacio pueda crearse se necesitan condiciones básicas compartidas por ambas partes, tales como confianza (Dubois y Karcher, 2005) y compromiso (Rízquez, 2008). El proceso a ocurrir en ese espacio "estará definido por los tipos de apoyo que el mentor proporciona al mentorando" (Eby et al., 2010, p. 10). Así, entre las acciones mencionadas por los mentorandos en el programa Talentum-Universidad, la que obtuvo un mayor número de menciones fue "que el mentor busque crear un ambiente de confianza". Otras acciones mencionadas con frecuencia fueron, "que muestre interés en lo que yo le estoy contando"; "que tenga apertura para compartir conocimientos o saberes", y "disposición para compartir experiencias".

En este espacio que representa la mentoría, los estudiantes buscan explorar ideas sobre temas de su interés y sobre sí mismos; y esperan también recibir diferentes tipos de apoyo. Fue posible observar como confluyen ámbitos de diferente naturaleza: futuro profesional, futuro académico y retroalimentación sobre habilidades propias. Esta confluencia fue documentada por Johnson (2006, como se cita en Rízquez, 2008, p. 63) quien afirma que "las relaciones de mentoría implican, por lo tanto, la provisión de apoyo profesional, social y emocional en un entorno seguro para la autoexploración que da como consecuencia resultados académicos y personales positivos para los estudiantes".

Como una alianza de aprendizaje externa al aula, la mentoría ayuda al estudiante a desarrollar habilidades y actitudes, y construcción de conocimientos. Eby et al. (2010) afirman que, independientemente de las metas a trabajar, de las locaciones, de calidad de las relaciones y de las expectativas de los participantes, toda mentoría involucra la adquisición de conocimiento.

Como demostró la evidencia, los estudiantes trabajan en la mentoría metas relacionadas con su futuro laboral y académico. En la edición más reciente del programa, el tipo de meta que superó a estas dos fue el interés de los estudiantes por recibir de su mentor retroalimentación sobre habilidades propias con la finalidad de aspirar a un perfil más competitivo. Esto comprueba que en la mentoría ocurre más de un estado de desarrollo, como lo indican Rhodes y Dubois (2008, p. 255): "Relaciones de mentoría bien establecidas pueden contribuir a resultados positivos en jóvenes mediante tres procesos de desarrollo que interactúan entre sí: socio-emocional, cognitivo y relacionados con la identidad propia".

La mentoría como alianza de aprendizaje ofrece otras contribuciones específicas que son tangibles como beneficios. De acuerdo con lo reportado por los mentores, los estudiantes lograron avanzar -aunque en menor medida en comparación con las metas preponderantes ya mencionadas- en el desarrollo de habilidades y actitudes, como: seguridad en sí mismos, autoconocimiento, claridad al establecer prioridades, enfoque, receptividad y apertura. Por su parte, los estudiantes reportaron como principales beneficios (en orden de importancia) los siguientes: 1) Mayor claridad sobre los temas tratados, 2)

\footnotetext{
${ }^{11}$ En la edición 2016. El 3\% restante no respondió.

12 En la edición 2016. El 8\% reporta un avance parcial y el 2\% (1 estudiante) respondió que no.
} 
Retroalimentación y consejos recibidos del mentor, 3) Mayor preparación para fortalecer sus habilidades o realizar actividades específicas, 4) Mayor seguridad en sí mismo y para tomar decisiones, y 5) Haber recibido orientación en proyectos específicos.

Esto coincide en lo reportado por Rawlings (2002, como se cita en Knippelmeyer y Torraco, 2007, p. 3):

(...) los mentorandos en las relaciones de mentoría a menudo experimentan una multitud de beneficios: mayor confianza en sí mismos; una mayor disponibilidad de asesoramiento e información relevante; una oportunidad para estimular la reflexión sobre la práctica; apoyo personal adicional; mejora de la eficacia; una conciencia de la cultura, la política y la filosofía de la organización; y el acceso a un confidente sobre preocupaciones o ideas.

Si bien excede el objetivo de esta investigación, existe evidencia a partir del caso estudiado que puede informar futuros estudios; por ejemplo, sobre el perfil del mentor, a partir de sus características y acciones realizadas más valoradas, como las reportadas por los participantes del programa investigado. Otro tema de estudio es la figura del mentor y los múltiples "roles de servicio" (Gehrke, 1998, p. 192) que estos llevan a cabo; así como posibles beneficios para los mentores (Alderman 2000, como se cita en Knippelmeyer y Torraco, 2007, p.5, y Gehrke 1998). De la experiencia de Talentum-Universidad se desprenden prácticas y aprendizajes para informar el diseño de políticas institucionales que impulsen la mentoría como una práctica que contribuye a la formación integral de estudiantes universitarios y que puede facilitarles la transición a posteriores etapas de desarrollo.

\section{Referencias}

Alderman, B. (2000). Mentoring relationships. Actas de la Australian Library and Information Association. Recuperado de http://conferences.alia.org/au/alia2000/proceedings/belle.alderman.html

Anderson, E. M. y Shannon, A. L. (1988). Toward a conceptualization of mentoring. Journal of Teacher Education, 39, 38-42.

Bozeman, B. y Feeney, M. K. (2007). Toward a useful theory of mentoring: a conceptual analysis and critique. Administration \& Society, 39, 719-739.

Burke, R. J. y Mckeen, C. A. (1997). Benefits of mentoring relationships among managerial and professional women: a cautionary tale. Journal of Vocational Behavior, 51(1), 43-57.

Clark, E. (1994). Mentoring: its potential for staff development and learning enhance-ment in a legal environment. Journal of Professional Legal Education, 12, 239.

Coles, A. (primavera, 2011). The role of mentoring in college access and success. Research to Practice Brief, $1-10$.

DuBois, D. L. y Karcher, M. J. (2005). Youth mentoring: theory, research and practice. En D. L. DuBois y M. J. Karcher (Eds.), Handbook of youth mentoring (pp. 2-12). Thousand Oaks, CA: Sage.

Eby, L. T., Rhodes, J. E. y Allen, T. D. (2010). Definition and evolution of mentoring. En T. D. Allen y T. L. Eby (Eds.), The blackwell handbook of mentoring: a multiple perspectives approach (pp. 7-20). Nueva York: John Wiley \& Sons.

Garringer, M., Kupersmidt, J., Rhodes, J., Stelter, R. y Tai, T. (2015). Elements of effective practice for mentoring. (4th Edition). Boston, MA: MENTOR: The National Mentoring Partnership. Recuperado de http://www.mentoring.org/program-resources/elements-of-effective-practice-for-mentoring/ 
Gehrke, N. (1988). Toward a definition of mentoring. Theory into Practice, 27, 190-194.

Gibbons, A. (2000). Getting the most from mentoring: recent developments and learning. Training Journal -ELY-, 18-20. Recuperado de https://scholar.google.com.mx/scholar?cluster=14383950302765184117\&hl=es\&as sdt=0,5

Gulam, W. A. and Zulfiqar, M. (1998). Mentoring: Dr. Plum's elixir and the alchemist's stone'. Mentoring \& Tutoring, 5(3), 39-45.

Hall, J. C. (2003). Mentoring and young people: a literature review. The sCRE Centre, University of Glasgow. Recuperado de http://www.educationandemployers.org/wp-content/uploads/2014/06/ mentoring- and-young-people-hall.pdf

Healy, C. C. y Welchert, A. J. (1990). Mentoring relations: a definition to advance research and practice. Educational Researcher, 19(9), 17-21.

Jacobi, M. (1991). Mentoring and undergraduate academic success: a literature review. Review of Educational Research, 61(4), 505-532.

Johnson, A. W. (2006). An evaluation of the long-term impact of the Sponsor-a-Scholar (SAS) Program on student performance. Princeton, Nu: Mathematica Policy Research.

Johnson, C. S. (1989). Mentoring programs. En M. L. Upcraft y J. Gardner (Eds.), The freshman year experience: Helping students survive and succeed in college (pp. 118-128). San Francisco, CA: Jossey-Bass.

Knippelmeyer, S. A. y Torraco, R. J. (2007). Mentoring as a developmental tool for higher education. Documento presentado en la Academy of Human Resource Development International Research Conference in The Americas (Indianapolis, IN, Feb 28-Mar 4).

MENTOR/National Mentoring Partnership (2003). Elements of effective practice. Alexandria, vA.

O'Neill, K. y Harris et al. (2005). Perspectives on e-mentoring: a virtual panel holds an online dialogue. National Mentoring Center Newsletter, 9, 5-12.

Rhodes, J. E. (2002). Stand by me: the risks and rewards of mentoring today's youth. Cambridge, MA: Harvard University Press.

Rhodes, J. E. y Dubois, D. L. (2008). Mentoring relationships and programs for youth. Current Directions in Psychological Science, 17(4), 254-258.

Rísquez, A. (2008). E-mentoring: an extended practice, an emerging discipline. En F. J. García-Peñalvo (Ed.), Advances in e-learning: experiences and methodologies (pp. 61-82). Nueva York: Information Science Reference.

Roberts, A. (2000). Mentoring revisited: a phenomenological reading of the literature. Mentoring \& Tutoring: Partnership in Learning, 8(2), 145-170.

Yamamoto, K. (1988). To see life grow: The meaning of mentorship. Theory into Practice, 23(3), 183-189. 


\section{Anexo 1}

\section{Programa Talentum-Universidad (5 Etapas)}

1a. etapa (mes de marzo): Semana de inducción en la Ciudad de México. Los 60 estudiantes seleccionados participan en una agenda de trabajo que incluye: talleres, conferencias magistrales, actividades académicas y de integración, y charlas con invitados especiales del sector privado, público y de la sociedad civil.

2a. etapa (marzo a julio): Trabajo en equipo a distancia. Desde su lugar de residencia, los estudiantes trabajan en un proyecto en equipo asignado durante la primera semana de inducción. Cada 15 días envían a la coordinación del programa una entrega parcial (de un total de 7). Los equipos trabajan bajo la dirección de un facilitador, quien evalúa los avances y brinda retroalimentación a lo largo del proceso.

3a. etapa (mes de julio): Componente internacional. Viaje académico a EE.UU. Los estudiantes visitan universidades de alto prestigio para realizar actividades académicas como conferencias con especialistas, paneles con expertos en innovación, negocios y sustentabilidad. Así como visitas a sitios de interés locales como incubadoras de emprendimiento, centros de investigación y para el desarrollo de tecnología. Además, los estudiantes reciben retroalimentación respecto al proyecto que trabajaron en equipo.

4a. etapa (mes de julio, previo a la clausura): Práctica "sombra" con líderes. En la Ciudad de México, los estudiantes realizan una "práctica sombra" (shadowing, en inglés), que implica compartir una jornada laboral típica con líderes de la academia, del servicio público, de la iniciativa privada y de la sociedad civil.

5a. etapa (mes de julio): Presentación de proyecto en equipo y clausura del programa. Los estudiantes presentan ante un jurado calificador el proyecto que trabajaron en equipo y posterior a la premiación de los mejores trabajos, se lleva a cabo la clausura del programa 


\section{Anexo 2}

\section{Perfil de los integrantes del programa Talentum-Universidad}

2a. generación (Edición 2016)

> 60 participantes: 30 hombres y 30 mujeres.

$>44$ de universidades públicas; 16 de universidades privadas.

$>$ Edad: 22 años (promedio).

$>$ Procedencia: de 27 entidades federativas. Aguascalientes (4); Baja California (2); Campeche (1); Chiapas (3); Chihuahua (2); Colima (2); Ciudad de México (7); Durango (2); Estado de México (3); Guanajuato (1); Hidalgo (1); Jalisco (2); Michoacán (3); Morelos (1); Nayarit (1); Nuevo León (3); Puebla (3); Oaxaca (1); Querétaro (2); Quintana Roo (2); Sinaloa (2); Sonora (1); Tabasco (1); Tamaulipas (1); Veracruz (2); Yucatán (6); y Zacatecas (1).

$>$ Carreras: 40 de ingenierías y 20 de licenciaturas.

> Ingenierías: Industrial y de Sistemas (1); Industrial (1); Bioquímica (1); Mecatrónica (8); Aeroespacial (1); Energías Renovables (1); Procesos Alimentarios (1); Gestión Empresarial / Negocios (6); Informática (1); Mecánica (2); Petrolera (1); Negocios y Tecnologías de la Información (1); Geofísica (1); Civil (2); Financiera (1); Física Industrial (1); Tecnologías de la información / Ciencias de la Computa-ción (2); Química Industrial (2); Química (2); Sistemas (1); Seguridad Industrial y Automatización (1); y Química en Procesos Sustentables (2).

$>$ Licenciaturas: en Políticas Públicas/ Ciencia Política (4); Derecho (2); Pedagogía (1); Administración de Empresas (1); Tecnología (2); Trabajo Social (1); Comunicación (1); Mercadotecnia (1); Economía (1); Relaciones Internacionales y Comercio (1); Relaciones Internacionales (1); Mercadotecnia y Negocios Internacio-nales (1); Antropología Social (1); Conservación y Restauración de Patrimonio Cultural (1); Nutrición (1).

\section{1a. generación (Edición 2016)}

> 60 participantes: 30 hombres y 30 mujeres.

$>40$ de universidades públicas; 20 de universidades privadas.

$>$ Edad: 22.5 años (promedio).

$>$ Procedencia: de 20 entidades federativas. Ciudad de México (10); Estado de México (9); Querétaro (4); Veracruz (4); Yucatán (4); Aguascalientes (3); Jalisco (3); Michoacán (3); Nuevo León (3); Oaxaca (3); Puebla (3); Guanajuato (2); Tlaxcala (2); Chiapas (1); Chihuahua (1); Durango (1); Hidalgo (1); Morelos (1); Nayarit (1); y Sonora (1).

$>$ Carreras: 18 de ingenierías; 37 en licenciaturas y 5 en otras carreras.

> Ingenierías: (3) Sistemas Computacionales y o Computación; (2) Civil; (2) Industrial; (3) Electrónica; (1) en Tecnologías Computacionales; (1) en Automatización; (1) en Ambiental; (1) Biomédica; (1) Mecatrónica; (1) en Química Ambiental; (1) Mecánico Administrador; (1) Biotecnología.

> Licenciaturas: (4) Ciencia Política; (4) Política y Administración Pública; (4) Economía; (3) en Matemáticas; (3) Administración Financiera; (2) Administración; (2) Administración y Negocios internacionales; (2) Dirección y Administración de Empresas; (2) Relaciones Internacionales; (1) Comercio internacional; (1) Contaduría Pública y Finanzas; (1) Derecho; (1) Desarrollo de Negocios y Gestión Empresarial; (1) Enseñanza de Idiomas; (1) Física y Matemáticas; (1) Lenguas Extranjeras; (1) Letras Mexicanas; (1) Psicología; (1) Pedagogía; (1) Relaciones Comerciales.

> Otras carreras: (3) Químico Farmacéutico Biólogo; y (2) Arquitectura. 